\title{
Fast learning neural network based on texture for Arabic calligraphy identification
}

\author{
Ahmed Kawther Hussein \\ Department of Computer Science, College of Education, Mustansiriyah University, Baghdad, Iraq
}

\begin{tabular}{|c|c|}
\hline Article Info & ABSTRACT \\
\hline Article history: & Arabic calligraphy is considered a sort of Arabic writing art where letters in \\
\hline Received Jul 28, 2020 & Arabic can be written in various curvy or segments styles. The efforts of \\
\hline Received Jul 28, 2020 & automating the identification of Arabic calligraphy by using artificial \\
\hline Revised Oct 13, 2020 & intelligence were less comparing with other languages. Hence, this article \\
\hline Accepted Nov 11, 2020 & $\begin{array}{l}\text { proposes using four types of features and a single hidden layer neural } \\
\text { network for training on Arabic calligraphy and predicting the type of }\end{array}$ \\
\hline Keywords: & $\begin{array}{l}\text { calligraphy that is used. For neural networks, we compared the case of non- } \\
\text { connected input and output layers in extreme learning machine ELM and the }\end{array}$ \\
\hline $\begin{array}{l}\text { Arabic calligraphy } \\
\text { Binarized statistical image }\end{array}$ & $\begin{array}{l}\text { case of connected input-output layers in FLN. The prediction accuracy of fast } \\
\text { learning machine FLN was superior comparing ELM that showed a variation } \\
\text { in the obtained accuracy. }\end{array}$ \\
\hline
\end{tabular}

Local phase quantization
This is an open access article under the CC BY-SA license.

\begin{abstract}
Arabic can be written in various curvy or segments styles. The efforts of automating the identification of Arabic calligraphy by using artificial network for training on Arabic calligraphy and predicting the type of calligraphy that is used. For neural networks, we compared the case of noncase of connected input-output layers in FLN. The prediction accuracy of fast in the obtained accuracy.
\end{abstract}

\section{Corresponding Author:}

Ahmed Kawther Hussein

Department of Computer Science

College of Education

Mustansiriyah University, Baghdad, Iraq

Email: ahmedkawther@uomustansiriyah.edu.iq

\section{INTRODUCTION}

Arabic calligraphy is a unique way of writing Arabic using the style of curving the letters. The term used to indicate to Arabic calligraphy is Khat [1]. It is derived from the Arabic word 'line' as the line is the basic constructor in Arabic calligraphy [2]. It has many types such as Naskh, Diwani, Reqa, Thuluth ..etc. The oldest Arabic calligraphy type is Kufic where the style involves building rigid and angular strokes.

The fast-emerging of artificial intelligence AI and machine learning ML-based computer vision technologies has opened the door for a wide range of applications. The applications of AI and ML in computer vision include face recognition [3], ear recognition [4], medical diagnostic [5], autonomous driving [6], mammography [7], industrial application [8], diabetic retinopathy [9] and many other applications. Some of the interesting applications of ML-based computer vision are handwriting recognition [10] which involves identifying handwritten letters from the image. Also, writer identification [11] which involves recognizing the writer from his writing style. On the other side, It is observed that many other applications are also emerging day after day. This article is focusing on a special field of the area of ML-based models relating to handwriting in the Arabic language. This field is Arabic calligraphy identification using computer vision and machine learning. Unfortunately, few studies have focused on this field despite its good potential in various applications in technology for serving art and old manuscripts.

This article aims at building a novel framework for Arabic calligraphy identification using computer vision and machine learning. For computer vision, a set of texture features will be extracted from the image of Arabic calligraphy. For machine learning, two phases will be conducted. A separate fast learning neural 
network will be trained on each type of feature in the first phase and all neural networks will be fused together in the second phase. The remaining of the article is organized as follows. In Section 2, we present the literature survey. Next, the methodology is provided in Section 3. Afterward, the experimental works and results are provided in Section 4. Finally, the conclusion and future work are provided in Section 5.

\section{LITERATURE SURVEY}

The literature contains few attempts in exploiting or developing machine learning-based models for Arabic calligraphy [12] introduce the Auto encoder as a deep learning method used to recognizing Arabic calligraphy types, The Auto encoder has ability of reducing data dimensions in and extract features. In the work of [13], the authors have prepared a dataset of Arabic calligraphic letters along with a corresponding corpus of phrases and quotes. The letters dataset contains 3,457 images for a totally of 32 various categories of Arabic calligraphic type letters. The dataset was collected from a wide range of sources and they concluded from their article that combining such data with machine learning models is adequate for enabling machine learning to read and understand Arabic calligraphy. In the work of [14] set of texture features were used to analyze Arabic artistic style. The finding is that the best performance has been yielded by the BSIF descriptor with the SVM classifier [15] suppose a new approach for developing a method for generating Arabic handwriting by testing 7 types of Arabic calligraphy in the work of [16] the author proposed a new framework of optical font recognition for Arabic calligraphy by enhancing the binarization method the [17] present computational abstractions for generating and manipulating calligraphic compositions systematic by $\mathrm{s}$ within an interacting environment [18] suppose multi-classifier decisions as based off or Arabic-calligraphy style classification. The [19] used a new technique by define the three important coordinates in the image of each character and then translates it into triangle geometry style.

This article provides a novel system for identifying the style of Arabic Writing from a set of candidate styles or Arabic Calligraphy. It is the first developed machine learning model for predicting the type of used Arabic Calligraphy in a testing image. It extracts a set of texture features and trains a fast learning algorithm for this purpose. The novelty of this article is regarding combining eight types of texture features for the task of Arabic style identification. The combination is based on an aggregation rule. We name it as an aggregation based texture features trained fast learning neural network ATFLN.

\section{METHODOLOGY}

This section provides the developed methodology for Arabic Calligraphy identification. The features are provided in sub-section 3.1. Next, the fast learning network is given in subsection 3.2. Afterward, we present the aggregation rule in sub-section 3.3. The dataset is explained in sub-section 3.4. Lastly, the evaluation metrics are provided in sub-section 3.5.

\subsection{Texture features for Arabic calligraphy}

This section provides the texture features that will be extracted from an Arabic calligraphy photo. In subsection 3.1.1, we present local binary features LBP. Next, in subsection 3.1.2, we present local phase quantization LPQ features. Next, binarized statistical image features BSIF is provided in subsection 3.1.3. Next, pattern of oriented edge magnitude POSEM is presented in subsection 3.1.4. The used configurations of the features are provided in Table 1.

\subsubsection{Local binary pattern LBP}

LBP features are proposed initially by [20]. The original concept of LBP is to characterize the texture pattern of an image using $\mathbf{3} \times \mathbf{3}$ square neighborhoodthresholded by the value of the center pixel considering the sign information only which provides a local binary pattern. This is provided in the below;

$$
\begin{aligned}
L B P_{r, p} c & =\sum_{i=1}^{p-1} \operatorname{sign}\left(g_{i}-g_{c}\right) 2^{i} \\
\operatorname{sign}(x) & =\left\{\begin{array}{l}
1, x \geq 0 \\
0, x<0
\end{array}\right.
\end{aligned}
$$

\footnotetext{
$p$ the neighbor pixels

$g_{i}$ denotes the grey value of the pixel $i$

$g_{c}$ denotes the grey pixel of the center

$L B P_{r, p}{ }^{c}$ local binary pattern of center pixel ${ }^{c}$ with a radius $r$ and neighbor
} 
There are various types of LBP, we select uniform type, with a radius of 2 pixels, neighbor size $\mathbf{8}$

\subsubsection{Local phase quantization $L P Q$}

Local phase quantization LPQ is a type of feature based on the blur invariance property of the Fourier phase spectrum [21]. Such features are good to provide a practical discrimination capability and robustness to blurring effects. The concept of the features is summarized as following: 1- use a local window and calculate phase information of it. 2- use the phases of the four low-frequency coefficients for decorrelation and quantization in eight-dimensional space. 3- Build the histogram of the resulted code. Actually, this histogram is used as the features of LPQ. We use for extracting this features a window size of $5 \times 5$.

\subsubsection{Binarized statistical image features BSIF}

This method of feature extraction was proposed by [22]. The concept of this method is to build a binary code string for the image. It indicates to the local intensity pattern in the pixel neighbors. Next, a histogram of the pixels code is used to characterize the texture property. The steps are as follows: each bit is associated with a linear filter that is learned based on a training set of natural image patches by maximizing the statistical independence of the filter responses. Next, the response of linear filter is binarized using a threshold of zero. We point out that the number of features is determined based on the desired length of bit string. Finally, the histogram is built. For our feature extraction, we use number of filters equals to 8, filter size equal to $11 \times 11$, block size of $18 \times 18$.

\subsubsection{Pattern of oriented edge magnitude POEM}

This feature was proposed by [23]. Computing the POEM feature is based on the following steps: 1calculate the gradient of the image and perform a discretization over $0, \pi$ for unsigned representation and over $0,2 \pi$ for signed representation. 2- compute a histogram of gradient orientation overall cell pixels. 3encode the accumulated magnitude usingan LBP operator within a block. We use 3 orientation, uniform LBP with a radius of 2 and neighborhood size of 8 and block size of $12 \times 12$.

Table 1. Configuration of used texture features for our ATFLN

\begin{tabular}{cc}
\hline Feature Type & Feature Configurations \\
\hline LBP (citation) & Uniform, Radius: 2pixels, neighbour size:8, block size: 8X 8, no block overlap \\
LPQ (citation) & Window size: 5 X 5, block size 18 X 18, no overlap \\
BSIF & \# Filters : 8, filter size: 11 X 11, block size: 18 X 18, no overlap \\
POEM & \# Orientation: 3, uniform LBP, radius: 2 pixels, neighbourhood size: 8, block size: 12 X 12 pixels, no block overlap \\
\hline
\end{tabular}

\subsection{Fast learning neural network}

Fast learning neural network is one hidden layer neural network with connections between the input layer and hidden layer, connections between the hidden layer and the output layer, and connections between the input layer and output layer. It can be considered as a developed variant of extreme learning machine [24]. The training of this neural network is as follows.

a) Randomly initialize the weights in the input hidden layer.

b) Calculate the output matrix

$$
Y=f\left(W^{o i} X+W^{\text {oh }} G+C\right)=f\left(\left[\begin{array}{lll}
W^{o i} & W^{\text {oh }} & C
\end{array}\right]\left[\begin{array}{l}
X \\
G \\
I
\end{array}\right]\right)
$$

Where,

$W^{\text {oi }}$ denotes the input-output weights

$W^{\text {oh }}$ denotes the hidden output weights

$C$ denotes the biases

$X$ denotes the input features

Use the labeled data (training data) to calculate the weights for the input-output layer and the hidden layer output layer. 


$$
\vec{W}=f^{-1}(Y)\left(\begin{array}{l}
X \\
G \\
I
\end{array}\right)^{\dagger}
$$

$Y$ denotes the labels of the training data

We used FLN because it is shallow type of network which makes their training time shorter as well as their prediction time comparing with deep networks in addition that they have universal approximation power.

\subsection{Aggregation rules}

For the aggregation, we use the max-average filter. The concept of this filter is as follows. Considering that we have a set of $m$ classifiers: $C_{1}, C_{\mathbf{2}}, \ldots C_{m}$. Each is combined with $n$ elements where $n$ denotes the number of classes. Then $C_{i}$ is $\left(x_{i, 1}, x_{i, 2}, \ldots x_{i, n}\right)$. The max average prediction is based on averaging the values of the component $C_{i}$ and selecting deciding the class as the component with maximum value.

\subsection{Dataset}

The dataset is named as Printed Arabic Text Set or (PATS-A01) [25] is combined with a 2766 text line image that was selected from standard classic Arabic books. For this dataset, eight font were chosen: Arial, Tahoma, Akhbar, Thuluth, Naskh, Simplified Arabic, Andalus, and Traditional Arabic. We present two samples of the data in the Figure 1.

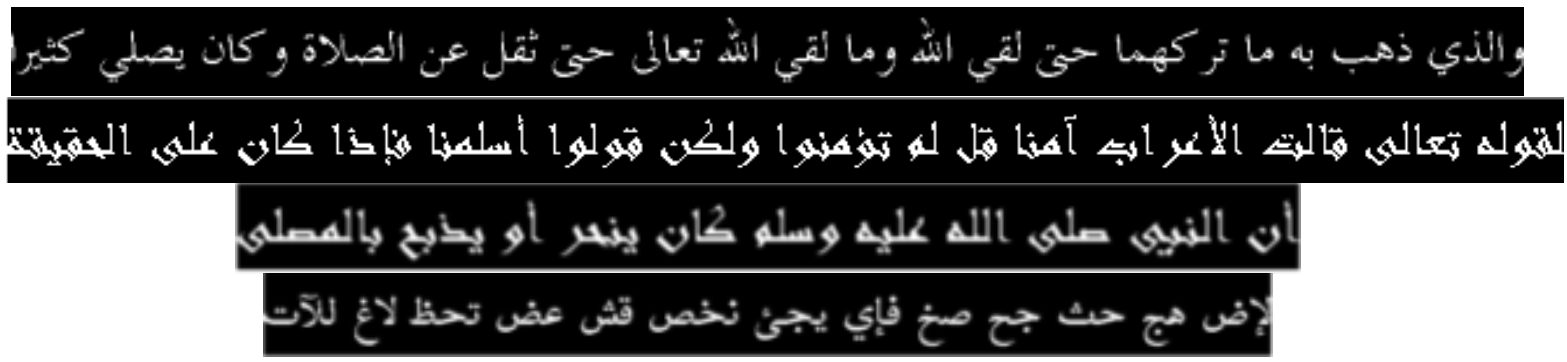

Figure 1. Two samples of PATS-A01

\subsection{Evaluation metrics}

For evaluation, we use the accuracy which is defined by the number of miss-classification over the total number of predictions. This measure is given by the below;

$$
\text { ACC }=\frac{\text { true predictions }}{\text { number of misclassifications }}
$$

\section{EXPERMENTIAL WORK}

In order to evaluate the proposed approach, we generate the accuracy of each of the proposed feature based on the presented dataset. We use two models: the first one is an extreme learning machine ELM [26] and the second one is a fast learning machine as it is depicted in Figures 2 and 3 respectively. The number of neurons has been changed from one neuron up to 10 neurons and in each experiment, the four types of features were used. The performance shows a change in the performance of ELM according to the type of feature and the number of hidden neurons while the accuracy of FLN was stable and equals to the value of $100 \%$ regardless of the number of neurons or the number of features. The interpretation of the high accuracy was achieved by FLN is that the training was conducted on samples that have not only the different types of Arabic calligraphy but different content of the text. This has enabled the neural network to identify the record. However, in ELM the fewer connections comparing with its equivalent of FLN has resulted in variation in the accuracy with having similar performance between the features and case of superiority based on POEM feature when 4 neurons were used. 


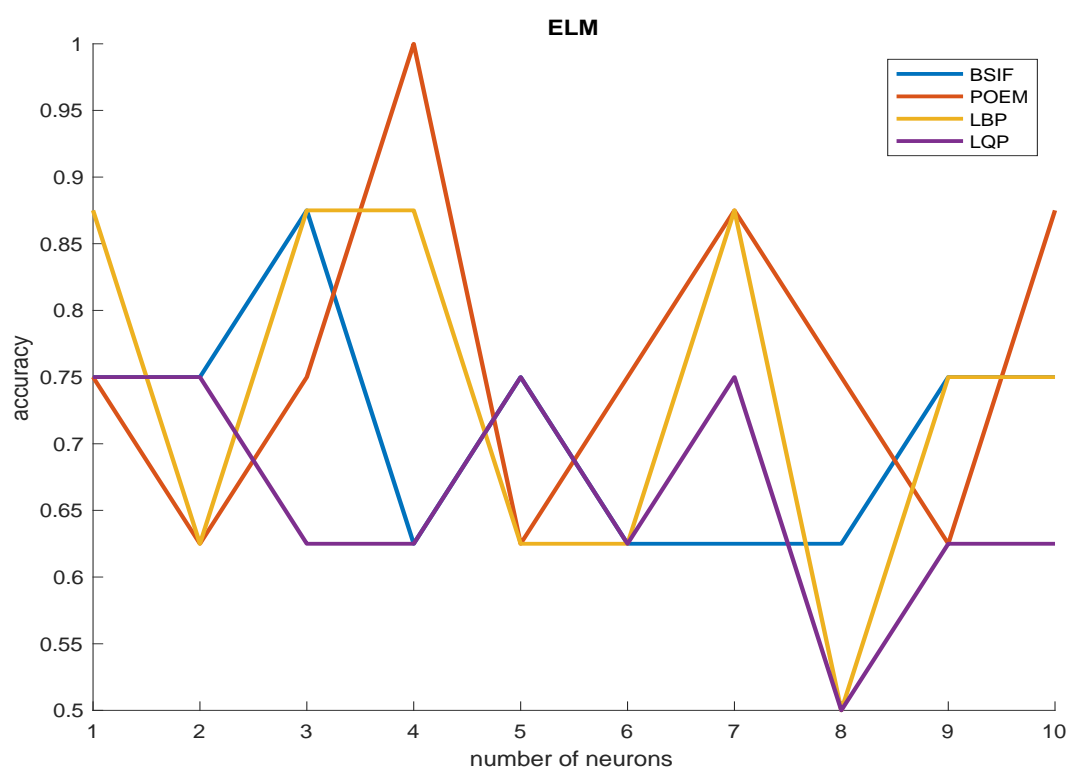

Figure 2. the test accuracy of ELM with different number of features and neurons

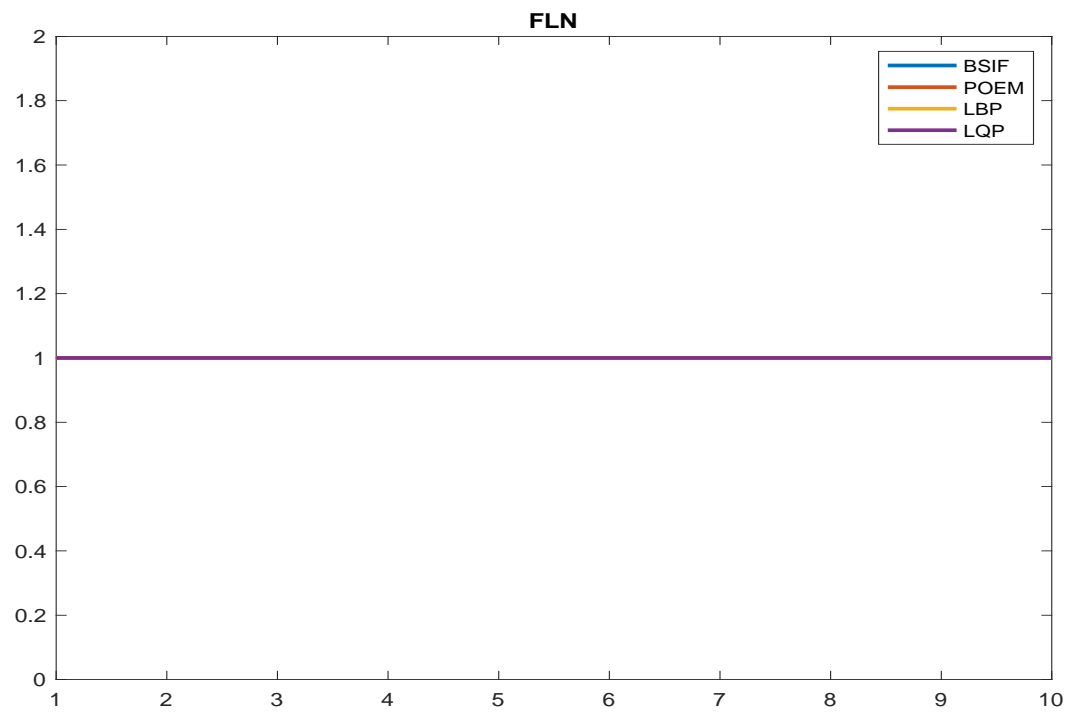

Figure 3. the test accuracy of FLN with different number of features and neurons

\section{COMCLUSION AND FUTURE WORK}

This article has presented an approach for detecting the type of Arabic calligraphy. It uses four types of features BSIF, POEM, LBP and LQP. It applied two types of neural networks, the first one is the extreme learning machine ELM and the second one is the fast learning machine FLN. The difference between both is the connections between the input and output layer which exists in FLN and it does not exist in ELM. The testing on Arabic calligraphy provides a superiority and stability of FLN regardless of the number of neurons. Another observation is the similarity in the performance of the features when ELM was used. Future work is to apply an ensemble learning of the proposed features and test the system on different types of writing styles in other languages.

\section{ACKNOWLEDGEMENTS}

The author would like to thank the Mustansiriyah university (www.uomustansiriyah.edu.iq) Baghdad-Iraq for its support in the present work. 


\section{REFERENCES}

[1] B. Bataineh, S. N. H. Sheikh Abdullah, and K. Omar, "Generating an arabic calligraphy text blocks for global texture analysis," International Journal on Advanced Science, Engineering and Information Technology, vol. 1, no. 2, pp. 150-155, 2011.

[2] M. S. Azmi, K. Omar, M. F. Nasrudin, A. K. Muda and A. Abdullah, "Arabic calligraphy classification using triangle model for Digital Jawi Paleography analysis," 2011 11th International Conference on Hybrid Intelligent Systems (HIS), Melacca, pp. 704-708, 2011, doi: 10.1109/HIS.2011.6122194.

[3] J. Deng, J. Guo, N. Xue and S. Zafeiriou, "ArcFace: Additive Angular Margin Loss for Deep Face Recognition," 2019 IEEE/CVF Conference on Computer Vision and Pattern Recognition (CVPR), Long Beach, CA, USA, pp. 4685-4694, 2019, doi: 10.1109/CVPR.2019.00482.

[4] M. Hassaballah, H.A. Alshazly and A.A. Ali, "Ear recognition using local binary patterns: A comparative experimental study. Expert Systems with Applications," vol. 118, pp. 182-200S, 2019.

[5] S. Razia and M.N. Rao, "Machine learning techniques for thyroid disease diagnosis-a review," Indian J Sci Technol, vol. 9, no. 28, pp. 1-9, 2016.

[6] A. K. Bhunia, A. Das, A. K. Bhunia, P. S. R. Kishore and P. P. Roy, "Handwriting Recognition in Low-Resource Scripts Using Adversarial Learning," 2019 IEEE/CVF Conference on Computer Vision and Pattern Recognition (CVPR), Long Beach, CA, USA, pp. 4762-4771, 2019, doi: 10.1109/CVPR.2019.00490.

[7] A. M. Wallace, "Industrial applications of computer vision since 1982," in IEE Proceedings E - Computers and Digital Techniques, vol. 135, no. 3, pp. 117-136, 1988, doi: 10.1049/ip-e.1988.0016.

[8] R.C. Gonzalez and R. Safabakhsh, "Computer vision techniques for industrial applications and robot control," Computer, no. 12, pp. 17-32, 1982.

[9] V. Bellemo et al., "Artificial intelligence screening for diabetic retinopathy: the real-world emerging application," Current Diabetes Reports, vol. 19, no. 9, pp. 72, 2019.

[10] J. Deng, J. Guo, N. Xue and S. Zafeiriou, "ArcFace: Additive Angular Margin Loss for Deep Face Recognition," 2019 IEEE/CVF Conference on Computer Vision and Pattern Recognition (CVPR), Long Beach, CA, USA, 2019, pp. 4685-4694, doi: 10.1109/CVPR.2019.00482.

[11] S. Chen, Y. Wang, C.T. Lin, W. Ding and Z. Cao, "Semi-supervised feature learning for improving writer identification," Information Sciences, vol. 482, pp. 156-170, 2019.

[12] B. Bataineh, S.N.H. Sheikh Abdullah and K. Omar, "Generating an arabic calligraphy text blocks for global texture analysis," International Journal on Advanced Science, Engineering and Information Technology, vol. 1, no. 2, pp. 150-155, 2011.

[13] S. A. Salamah and R. King, "Towards the Machine Reading of Arabic Calligraphy: A Letters Dataset and Corresponding Corpus of Text," 2018 IEEE 2nd International Workshop on Arabic and Derived Script Analysis and Recognition (ASAR), London, 2018, pp. 19-23, doi: 10.1109/ASAR.2018.8480228.

[14] Z. Kaoudja, B. Khaldi and M. L. Kherfi, "Arabic Artistic Script Style Identification Using Texture Descriptors.," 020 1st International Conference on Communications, Control Systems and Signal Processing (CCSSP), EL OUED, Algeria, pp. 113-118, 2020, doi: 10.1109/CCSSP49278.2020.9151569.

[15] C.J. Vyborny and M.L. Giger, "Computer vision and artificial intelligence in mammography," AJR (American journal of roentgenology), vol. 162, no. 3, pp. 699-708, 1994.

[16] H.A. Al-Muhtaseb, S.A. Mahmoud and R.S. Qahwaji, "Recognition of off-line printed Arabic text using Hidden Markov Models," Signal processing, vol. 88, no. 12, pp. 2902-2912, 2008.

[17] B. Bataineh, S. N. H. S. Abdullah and K. Omar, "Arabic calligraphy recognition based on binarization methods and degraded images," 2011 International Conference on Pattern Analysis and Intelligence Robotics, Putrajaya, 2011, pp. 65-70, doi: 10.1109/ICPAIR.2011.5976913.

[18] H. Moustapha and R. Krishnamurti, "Arabic calligraphy: A computational exploration," In 3rd International Conference on Mathematics and Design, pp. 294-306, 2001.

[19] Z. Kaoudja, M. L. Kherfi and B. Khaldi, "An efficient multiple-classifier system for Arabic calligraphy style recognition," 2019 International Conference on Networking and Advanced Systems (ICNAS), Annaba, Algeria, 2019, pp. 1-5, doi: 10.1109/ICNAS.2019.8807829.

[20] T. Ojala, M. Pietikainen and D. Harwood, "Performance evaluation of texture measures with classification based on Kullback discrimination of distributions," Proceedings of 12th International Conference on Pattern Recognition, Jerusalem, Israel, 1994, pp. 582-585 vol.1, doi: 10.1109/ICPR.1994.576366.

[21] E. Rahtu, J. Heikkilä, V. Ojansivu and T. Ahonen, "Local phase quantization for blur-insensitive image analysis," Image and Vision Computing, vol. 30, no. 8, pp. 501-512, 2012.

[22] J. Kannala and E. Rahtu, "BSIF: Binarized statistical image features," Proceedings of the 21st International Conference on Pattern Recognition (ICPR2012), Tsukuba, 2012, pp. 1363-1366.

[23] N. S. Vu, and A. Caplier, "Face recognition with patterns of oriented edge magnitudes," In European conference on computer vision, pp. 313-326, Springer, Berlin, Heidelberg, 2010, September.

[24] Ali, Mohammed Hasan, Bahaa Abbas Dawood Al Mohammed, Alyani Ismail and Mohamad Fadli Zolkipli, "A new intrusion detection system based on fast learning network and particle swarm optimization," IEEE Access, vol. 6, pp. 20255-20261, 2018.

[25] H. Dawood, H. Dawood, and P. Guo, "Improved Arabic Word Classification using Spatial Pyramid Matching Method," Conference: International Conference Image and Vision Computing New ZealandI, 2011.

[26] A. K. Hussein, "Subject Review: Machine Learning and Deep Learning based Arabic Handwriting Recognition," International Journal of Engineering Research and Advanced Technology-IJERAT (ISSN: 2454-6135), vol. 5, no. 10, pp. 09-14, 2019. 